\title{
The influence of therapists' first-hand experience with psychedelics on psychedelic-assisted psychotherapy research and therapist training
}

\author{
ELIZABETH M. NIELSON* and JEFFREY GUSS
}

Department of Psychiatry, NYU School of Medicine, New York, NY, USA

(Received: May 19, 2018; accepted: July 26, 2018)

\begin{abstract}
Clinical research on psychedelic-assisted psychotherapy is rapidly advancing in the USA, with two drugs, psilocybin and MDMA, progressing through a structure of FDA-approved trials on a trajectory toward Drug Enforcement Agency rescheduling for therapeutic use. Researcher's and clinician's personal use of psychedelics was cited as a potential confound in psychedelic research studies conducted in the 1950s and 1960s, a concern which contributed to the cessation of this research for some 20 years. Currently, there is no empirical research on personal use of psychedelics by current academic researchers and clinicians; its influence is undocumented, unknown, and undertheorized. This paper explores the history of personal use of psychedelics by clinicians and researchers, the potential impact of personal use on psychedelic-assisted psychotherapy and research, and the rationale for opening an academic discussion and program of research to investigate the role of personal use. We propose that there are factors unique to psychedelic-assisted therapy such that training for it cannot neatly fit into the framework of modern psychopharmacology training, nor be fully analogous to psychotherapy training in contemporary psychological and psychiatric settings. We argue that scientific exploration of the influence of therapists' first-hand experience of psychedelics on psychedelic-assisted therapy outcomes is feasible, timely, and necessary for the future of clinical research.
\end{abstract}

Keywords: psychedelic therapists, psychotherapy training, psilocybin, MDMA, LSD, ethics

\section{INTRODUCTION}

Self-experimentation with psychedelic compounds by researchers and therapists played an important and largely undocumented role in the psychedelic therapy and research of the European and North American psychiatric mainstream from the 1950s through the early 1970s. Often cited by researchers as the very source of inspiration to study psychedelics in the first place, there was a substantial concern that the first-hand experience had contaminated the objectivity of the researchers (Mangini, 1998). Academically sanctioned research and clinical work with psychedelics, sometimes referred to as above-ground work, were disrupted by increasingly restrictive laws that emerged in the mid-1960s and culminated in the passage of the Controlled Substances Act of 1970. These laws were created in response to the warning regarding the use of psychedelics in recreational and non-medical settings and criminalized use of psychedelics outside of sanctioned research settings and placed stringent restrictions on their use in sanctioned research settings. Although research on psychedelic-assisted psychotherapy was technically still legal and possible, a second trend toward increasing focus on double-blind trial design, for which psychedelicassisted therapy was a poor fit, coincided with the changing drug laws such that the research was effectively stopped for some 20 years (Oram, 2012). The influence and centrality of psychedelic therapists' and researchers' personal experience with psychedelics was a controversial point and consensus on the topic was not reached.

Sanctioned research on the effects of psychedelics in humans was reinitiated in 1990 by Strassman (2001), who studied the effects of intravenous dimethyltryptamine (DMT) on healthy volunteers at the University of New Mexico. Numerous FDA-approved clinical trials followed and we are now 25 years into the second wave of academic psychedelic research; however, the influence of first-hand experience with psychedelics on those who conduct clinical research and therapy with them remains underexplored and undertheorized. With this paper, we seek to open an academic dialogue on the role of researchers' and clinicians' personal experience with psychedelic compounds (be it as part of a training program, religious/shamanic ceremony, alone, or with a peer group) by asking what may be the impact of this experience on therapeutic outcomes. We propose that this should now be an askable and researchable question, and as such it should be moved from theoretical debate to a subject of formal inquiry. We will explore the history of personal use of psychedelics by researchers and psychotherapists who work with these compounds and then focus on the role of personal experience in therapist preparation and training among contemporary therapist

* Corresponding author: Elizabeth M. Nielson; Department of Psychiatry, NYU School of Medicine, New York, NY, USA; E-mail: elizabeth.nielson@nyumc.org

This is an open-access article distributed under the terms of the Creative Commons Attribution-NonCommercial 4.0 International License, which permits unrestricted use, distribution, and reproduction in any medium for non-commercial purposes, provided the original author and source are credited, a link to the CC License is provided, and changes - if any - are indicated. 
training programs. The parallels and divergences from psychoanalytic training and mindfulness-based interventions will be a part of our discussion.

\section{BACKGROUND: WHAT IS PSYCHEDELIC- ASSISTED PSYCHOTHERAPY?}

Following the discovery of the psychoactive effects of lysergic acid diethylamide (LSD) by Albert Hofmann in 1943, the compound was made available by Sandoz Pharmaceuticals to researchers with two explicit indications: to "elicit release of repressed material and provide mental relaxation" in psychotherapy and for psychiatrists to take in order to "gain an insight into the world of ideas and sensations of mental patients" (Hofmann, 2005, p. 73). Clinicians also obtained LSD and used it in psychotherapy and alcoholism treatment outside of research settings, a practice that was legal in the USA until the mid-1960s (Abramson, 1976). When the above-ground clinical and research work came to a halt, therapeutic work and research with psychedelic compounds did not end, instead it continued outside of mainstream institutions and without government approval or oversight (Nickles, 2015; Sessa \& Fischer, 2016). A full discussion of this underground work is outside our present inquiry, but we do not dismiss the relevance of these endeavors in any way.

\section{PSYCHEDELIC-ASSISTED PSYCHOTHERAPY AND PSYCHOLYTIC PSYCHOTHERAPY: DISTINCTION AND OVERLAP}

Psychedelic-assisted psychotherapy, as practiced in contemporary academic research settings, involves the use of a psychedelic compound in the context of a short-term (timelimited) sequence of therapy sessions that are structured to inform, shape, and interpret the psychedelic experience. Most current studies employ a sequence of three types of sessions: preparatory, medication, and integration sessions (Bogenschutz \& Forcehimes, 2016). These three parts prepare patients for the psychedelic sessions and establish the therapeutic alliance, to guide them safely during the psychedelic experience itself and to facilitate the process of translating that experience into meaningful long-term change. Psychedelicassisted psychotherapy generally uses medium to high doses of a psychedelic during 1-3 sessions that are spaced several weeks apart, with the intention for the patient to undergo a highly intense autobiographical and/or mystical experience, which is then explored during integration.

Psycholytic psychotherapy, in contrast, involves a lower dose of a psychedelic administered (at regular intervals or with varying frequency) during traditional psychotherapy sessions. The therapist employs active psychotherapeutic interventions, such as reflections and interpretations, while the patient is under the influence of the psychedelic. The reasoning behind this method is that the psychedelic loosens the patient's defenses and opens up unconscious affect and memory so that psychotherapy can be more effective, and hence the name psycholytic (loosening). This type of treatment is conducted in the context of an ongoing (medium- or long-term) psychotherapy (Walsh \& Grob, 2006). In practice these approaches are sometimes blended. For instance, therapists may encourage a more introspective approach during the highest intensity hours of a psilocybin session, but begin discussing the patients experience before the effects of the psychedelic compound have completely worn off.

Both psychedelic-assisted and psycholytic psychotherapy are designed to treat a specific malady or diagnosis. This distinguishes them from psychedelic sessions that have nonillness-related intentions, such as those undertaken for personal or spiritual growth. Most contemporary psychedelic research has focused on cancer-related distress (Griffiths et al., 2016; Grob et al., 2011; Ross et al., 2016), addiction (Bogenschutz et al., 2015; Johnson, Garcia-Romeu, Cosimano, \& Griffiths, 2014), or post-traumatic stress disorder (PTSD; Mithoefer, Wagner, Mithoefer, Jerome, \& Doblin, 2011). Psychedelic-assisted psychotherapy, as described above, is an approach presently being studied in the majority of large-scale research studies in academic settings. Although much of what we discuss will apply to therapists conducting psycholytic psychotherapy, we focus our discussion on research and findings that are relevant to psychedelic-assisted psychotherapy. We henceforth refer to psychedelic-assisted psychotherapy simply as psychedelic therapy and to those who provide it as psychedelic therapists. Researchers working in psychedelic research may also be psychedelic therapists, if they perform a clinical role on a research project, and we use the term psychedelic researchers to include non-clinician investigators and research support staff who may or may not interact with patient populations in their roles. Although portions of our discussion may also apply to these non-clinician researchers, here we focus on those whose roles include providing psychedelic therapy.

\section{The authors' relationship to psychedelic therapy}

As noted, psychedelic therapists and researchers who work in this field have previously been questioned regarding their own use of psychedelics, with the concern that direct experience might compromise their objectivities regarding their research, or that the absence of direct experience might compromise their effectiveness, even their validity, as therapists. For reasons outlined herein, we do not disclose our personal first-hand experience with psychedelics; however, given the sensitive nature of our topic, a discussion of our own roles and orientation to psychedelic research is fitting. Both authors are presently therapists in the ongoing psilocybin-assisted treatment of alcohol dependence trial at New York University School of Medicine (NYUSoM). JG was a therapist in the now-completed trial of psilocybinassisted treatment of cancer anxiety, also at NYUSoM (Ross et al., 2016). Additionally, he ran the psychedelic therapy training program for the 16 therapists who worked in the cancer anxiety trial, including mentorship and didactic sessions. Both authors have participated in the Multidisciplinary Association for Psychedelic Studies (MAPS)-sponsored 3,4,-methylenedioxymethamphetamine (MDMA)-assisted therapist training program and have served as therapists with the MAPS-sponsored trials of MDMA-assisted treatment of PTSD at NYUSoM. Currently, both authors are also part of a team conducting a qualitative interview study of therapists who provide psilocybin-assisted therapy in research settings. 
This paper grew out of discussions held during the design of that study regarding the safety, relevance, and importance of asking psychedelic therapists about personal experience with psychedelic compounds. In effect, the authors are both the inquirers and subject of inquiry for this paper, having carefully thought about material from both standpoints.

\section{Psychedelic compounds in the context of psychedelic therapy}

The compounds currently in use in psychedelic therapy studies vary considerably in their neurochemical effects; the choice of one psychedelic compound over another for a research study is affected by the feasibility of use, legal restrictions, neuroscience-based considerations, and even the cultural stigma associated with certain compounds. The weight of these factors varies among different countries in which the research is being conducted. Additionally, phenomenological effects of different drugs (and/or drug classes) vary considerably, and these variations may guide the selection and development of an optimal psychotherapy platform. A comprehensive discussion of psychedelic compounds is outside the scope of this paper; readers are referred to the recent review by Garcia-Romeu, Kersgaard, and Addy (2016). Our present discussion focuses on psychedelic therapy using psilocybin and MDMA, as these two compounds are currently the most commonly used ones in academic research settings in the USA.

Looking back to the early psychedelic therapy, in both research and clinical settings, we see variations in

- the degree to which preparation and integration sessions were emphasized,

- the psychedelic compound and dosage administered,

- the number and frequency of psychedelic sessions,

- the nature of the alteration in consciousness sought, and

- the methods used to guide the psychedelic sessions and preparation/integration sessions.

Likewise, we see variation in emphasis among these variables in contemporary clinical research protocols. These choices may reflect the backgrounds and beliefs of the researchers as well as the hypotheses of specific research project; they may also reflect an attempt to tailor treatment to the specific compound that is being studied. The following is an example of this variance in methodology: a recent study of ayahuasca (which contains DMT, a classic hallucinogen in the same category as psilocybin) for the treatment of major depressive disorder was restricted to pre- and post-treatment testing of psychiatric symptoms flanking a single ayahuasca session; no psychotherapy or integration sessions of any kind were provided (Osório et al., 2015). This contrasts with the work of the NYUSoM research team studying psilocybin-assisted treatment for alcohol dependence in which participants receive a series of psychotherapy sessions consistent with the evidencebased practice of motivational interviewing for alcohol-use disorders, along with sessions more directly focused on integrative work related to the psychedelic sessions (Bogenschutz et al., 2015).
HISTORY OF DIRECT EXPERIENCE THROUGH SELF-ADMINISTRATION BY PSYCHEDELIC RESEARCHERS AND THERAPISTS

Interest in psychedelic therapy rapidly grew after the discovery of LSD's psychoactive properties in 1943 and Sandoz's production, distribution, and program to supply LSD to researchers and clinicians (Hofmann, 2005). Some researchers and clinicians during this era stressed the value of direct experience with a psychedelic compound in order to function successfully as a psychedelic researcher and psychedelic therapist in particular (Blewett \& Chwelos, 1959; Leary, Metzner, \& Alpert, 2000). The idea, which Hofmann (2005, p. 76) wrote, was that the first-hand experience would "provide the doctors with direct insight, based on firsthand experience into the strange world of LSD inebriation, and make it possible for them to truly understand these phenomena in their patients, to interpret them properly, and to take full advantage of them." This experience was thought to engender compassion, understanding, and insight into the lived experience of psychosis, as well as that of patients undergoing an LSD session.

Some early US-based researcher/clinicians disclosed their personal use of LSD as a potentially relevant factor in their preparation to provide psychedelic therapy (Frederking, 1955; Smart, Storm, Baker, \& Solursh, 1966). Recent retrospective research has found that psychedelic researchers in the former Czechoslovakia had also found value in their personal use of LSD when conducting research between the 1950s and 1974, while it was prohibited there (Winkler \& Csémy, 2014; Winkler, Gorman, \& Kočárová, 2016). Mangini (1998) documented that among early psychedelic therapists, those who had not taken psychedelics themselves were considered by colleagues and critics to carry skeptical or uninformed attitudes toward the psychedelic experience, and that some writers speculated that these attitudes negatively influenced their patients' outcomes (Oram, 2012). Despite many arguments for and against such experience, the relationship between therapists' personal experience of psychedelics and patient outcomes remains unknown as quantitative empirical study has not yet been conducted.

In the psychotherapy literature, clinician-specific extrapharmacological factors, such as personal experience in relationships with individuals of other racial or ethnic backgrounds, and personal resilience and competence have been empirically tested and were found to influence the psychotherapy treatment outcomes (Green, Barkham, Kellett, \& Saxon, 2014; Larrison, Schoppelrey, Hack-Ritzo, \& Korr, 2011). In psychedelic therapy literature, the study of extrapharmacological influences other than the therapists' direct experience with psychedelic compounds on outcomes of psychedelic therapy has been explored theoretically (Hartogsohn, 2016) and empirically, albeit not recently, by Hyde (1960). Although not to contemporary standards of empirical research, Hyde tested extrapharmacological influences in a single-blind study by varying staff's behavior toward patients who are undergoing psychedelic therapy and found measurable increases in the negative effects of LSD, when patients were assigned to a treatment condition with less friendly staff and therapists. 
The field of contemporary psychiatry currently maintains that the direct experience of a psychotropic medication is neither necessary nor detrimental in effectively treating patients with that medication. Unfortunately, this area has not been explored in the literature: as of June 2018, we were unable to locate a single study on the relationship between psychiatrists' personal use of pharmaceutical substances, their prescribing practices with psychotropic medicines, and/or effects on patient outcomes. This scotoma was rendered more starkly for psychedelic therapy research with the emergence of randomized controlled trials (RCTs), which isolate treatments from the human beings who prescribe them or receive them. In the mid-1960s, RCTs became the gold-standard for efficacy of pharmacological treatments demonstrating direct biological link between the medicine and the pathology being treated (as in the case of antibiotics). Psychiatric medicines, psychotherapy, and psychedelic therapy in particular (which combines a pharmacological treatment with a psychotherapeutic intervention) have been poor fits for RCT research methods (Oram, 2012).

The idea that the researchers' and clinicians' first-hand experience with psychedelics could impact objectivity and ethical conduct of their work represents a conflict between two paradigms of research. On one hand, contemporary psychopharmacologic research values scientific objectivity in which personal experience is irrelevant and excluded as a source of knowledge (no one asks if those doing research with selective serotonin reuptake inhibitors (SSRIs) either take them or do not take them). On the other hand, in psychoanalytic research and training, subjective personal experience of the method is a valuable and respected source of information that may inform research, without risk of invalidating it. Controversy regarding the relevance, importance, and danger of self-experimentation in the current psychedelic research emerges from this dual nature: psychedelic therapy is an unprecedented blend of pharmacological and psychotherapeutic approaches that, as we will later discuss, do not neatly fit into one or the other category, carrying elements of both.

Regardless of one's position on the question of objectivity, we can confidently state that variation in therapists' personal experience with LSD and psilocybin introduces a potential confound to research efforts to demonstrate the efficacy of psychedelic therapy in a rigorous way. The nature of this confound is, in fact, an unaddressed empirical question: no contemporary studies have systematically studied whether or how therapists' first-hand experience with psychedelics affects clinical outcomes in psychedelic therapy. In the mid-1960s, researcher Herb Kleber came closest to doing so, when he designed a study to compare the outcomes of patients treated with LSD by a therapist who had not used LSD themselves versus one who had (with himself being the therapist in the study and taking his first dose of LSD between treating the two comparison groups). Unfortunately, the recall of LSD interrupted his work and the study was not completed (White, 2013). Even though it is methodologically quite complicated, empirical exploration of these questions is vital, and that question is rendered outside the scope of inquiry, if academic psychedelic research remains wholly embedded in the epistemology of objective psychopharmacologic research.

\section{Psychedelic experience as a part of psychedelic therapist training}

It is likely that the personal experience of academic-based psychedelic therapists spans a continuum, from those who are psychedelic naive to those who have extensive experience with a variety of compounds in diverse settings. A significant complication in this discourse is the illicit nature of psychedelics, at least in the USA, where the therapists are not free to speak openly about their levels of experience for the fear of professional consequences as individuals and in terms of the study as an entity. Given the history and contemporary legal status of psychedelics, therapists' use of them is an obvious potential target for attack. Therapists who have had the opportunity to take psychedelics in places where they are legal may feel somewhat more at ease discussing these experiences publicly, but there remains the threat of stigma and of having one's work discredited due to the potential confounding factors described above.

In the USA, since the restriction of access to psychedelic substances, only two studies have given psychedelic therapists the opportunity to take psychedelic medicines as part of their training: the Maryland State Psychiatric Institute group (during the 1970s, with LSD) and MAPS protocol known as MT-1, through which therapists' training to provide MDMA-assisted treatment of PTSD may elect to have a direct experience with MDMA. We will discuss each of these below. There is currently no similar program for therapists working with psilocybin (or any other psychedelic compound in the same family) to prepare them to work in the numerous active research initiatives that are underway in the USA.

\section{Spring Grove Research Center}

In 1969, researchers at the Spring Grove Research Center of the Maryland State Psychiatric Institute responded to numerous requests from therapists seeking an opportunity to undergo psychedelic-assisted therapy for training purposes. They applied to FDA to amend their existing LSD research protocols to include the administration of LSD to both therapists and researchers as a training experience (Kurland, Savage, Pahnke, Unger, \& Grof, 1969). Permission was granted; over 100 mental health professionals took up to three high doses of LSD as part of this program between 1969 and 1976 [Grof (2008) reported over 100 study participants between 1970 and 1974, whereas Yensen and Dryer (1992) reported 203 study participants between 1969 and 1976. Primary documents for this study were stored at the University of Purdue Library including files numbered $1-119$, with gaps at $77,81,109,115,117$, and 118 . It is unknown if there were additional files that were lost or destroyed.] (Grof, 2008; Yensen \& Dryer, 1992). Participants in this study were clinical researchers, therapists working with non-psychedelic-related approaches, and crisis interventionists, in addition to the psychedelic therapists in training. Follow-up data were collected but were never systematically analyzed and published, so our knowledge of outcomes remains anecdotal (Grof, 2008). An informal survey of three randomly selected files from the study reveals several instances of participants emphasizing the 
value of the experience in their professional and personal lives. At present, EMN is conducting an organized review of the study records to determine if and how participants benefited from the experience.

\section{MAPS' $M T-1$}

While prior experience with MDMA is not required for MDMA therapists, the sponsor for the ongoing trials of MDMA-assisted treatment of PTSD, MAPS, received FDA approval to administer MDMA to therapists who are to provide MDMA-assisted therapy for PTSD to participants in their studies (MAPS, 2010). This study, which is ongoing and based in part on the Spring Grove trial, is not designed to investigate the effect of the MDMA experience on clinicians' work as MDMA therapists, but rather to provide the opportunity for therapists in training to experience MDMA in a therapeutic setting, while collecting safety data in healthy volunteers (MAPS, 2010). Participants have, however, published their own accounts discussing the professional relevance of the experience. One wrote "My complete immersion in the session allowed me to walk away with a clearer image of how to be present with and for a client during their experience, and I felt more prepared to respond to what I would encounter as a therapist in an MDMA study" (Halberstadt, 2014, p. 5). A detailed analysis of how therapists undergoing the MDMA experience might incur such presence and preparedness has not been conducted.

\section{THE IMPORTANCE OF PERSONAL EXPERIENCE WITH ONE'S THERAPEUTIC METHOD}

Due to the protean nature of the psychedelic experience, and its well-known sensitivity to influence by set and setting (Hartogsohn, 2016), the skillful psychedelic therapist needs to approach the patient with certain clinical positions in place. These include a self-aware and non-judgmental attitude regarding the content and processes that emerge during sessions, valuing the aspects of experience that are beyond conventional notions of the self (i.e., states of ego dissolution, mystical states, and emotional flooding), a radical acceptance of highly emotional states and disordered thought (transient psychosis), sometimes a confidence in containing chaotic and transient changes in ego functioning, and a coherent stance regarding the relationship of this experience to integrative work. This is quite distinct from a traditional psychopharmacology model, in which the patient's diligent compliance with the prescribed regimen alone is expected to predictably reduce painful symptoms and improve functioning. In our current restrictive environment, and because of lack of empirical research on this topic, it is a challenge to know whether and how direct experience is relevant to offering safe and effective psychedelic therapy.

Strassman (2001) states that in Europe it is common, and even required, in some countries, that clinicians or researchers have first-hand experience with a psychedelic medicine before sitting with patients as a psychedelic therapist. He encourages the US investigators to consider implementing this practice to improve safety and allow for true informed consent. Furthermore, Strassman notes that this practice was also common in the USA prior to the 1970 restrictions on psychedelic use and research. Researchers and clinicians leading the current renaissance in psychedelic therapy training are acutely aware of the absence of open discussion regarding personal experience with psychedelics; however, addressing this topic is both complex and challenging. Given the absence of academic research on the role of psychedelic use by psychedelic researchers and therapists, we will discuss two examples of healing methods that must be experienced by providers from which we may draw some inferences.

\section{Potential parallel \#1: Training of healers who use psychedelics in religious/ritual settings}

Extensive experience with psychedelic compounds is a central aspect of training for shamans who work with them for diagnosis and treatment of a variety of ailments in ceremonial settings (Fernandez \& Fernandez, 2001; Winkelman, 2007). Worldwide there are numerous examples of shamanic traditions that incorporate psychedelic plants, and these examples may inform our discussion. In African Bwiti ceremonies, newcomers or ill persons may be given a high dose of ibogaine, whereas ceremony leaders and other participants, who previously initiated through multiple ibogaine rituals, take lower doses to join with the initiate. They maintain alertness through the night, while having aspects of a shared state of mind (Fernandez \& Fernandez, 2001).

Another example, ayahuasca, is a traditional psychoactive tea made by indigenous Amazonian tribes from plants that contain DMT and monoamine oxidase inhibitors. To become an ayahuascero (ayahuasca shaman) in traditional settings requires an apprenticeship that involves extensive ingestion of ayahuasca in a ritual context, under the guidance of a senior shaman, over a number of years (Thomas \& Humphrey, 1996, p. 93). In response to growing interest in ayahuasca, training institutes such as the Ayahuasca Foundation in Iquitos, Peru offer 6- and 10-week courses, which are "a condensed version of an ancestral initiation," and include three ayahuasca ceremonies per week as a part of a comprehensive training in the Shipibo tradition (Ayahuasca Foundation, 2017). Thus, any graduate will have substantial first-hand experience toward the end of the apprenticeship, will undertake leading ceremony under the guidance of the senior shaman, and will gradually become recognized in the community as a true and trusted ayahuascero or ayahuascera.

Although these examples could be used as model for training of psychedelic therapists in academic settings, therapeutic use of psychedelics in shamanic settings is based on a markedly different set of beliefs than academic psychedelic therapy (Metzner, 1998), and it remains unclear that how shamanic principles and practices might relate to the practices of academic psychedelic therapists. While we have no empirical evidence to demonstrate that indigenous cultures' practices regarding the training of psychedelic healers has relevance to contemporary academic research methods, there is ample a priori evidence that this is a question well worth asking, and important to keep at a central place in defining best practices for training therapists. 
Potential parallel \#2: Training for other psychotherapeutic interventions that evoke an alternative state of consciousness: Psychoanalysis and Mindfulness

Psychoanalysis and mindfulness-based interventions bear important similarities to psychedelic therapy. To begin, each of these is a method designed to evoke an alternative state of consciousness to facilitate access to normally unavailable and inaccessible parts of the self. Second, all three are predicated on the belief that the tools for healing are inherent in the individual's mind and largely outside the control of the therapist, but need some shape and direction (Freud, 1912; McCown, Reibel, \& Micozzi, 2010; Walsh \& Grob, 2006). Third, in both psychoanalysis and mindfulness-based interventions, the therapist is taught to use their own personal moment-to-moment experience in the room to understand and guide the patient and the process (Kabat-Zinn, 2006; Rubin, 1985). This type of presence is also cited by psychedelic therapists as essential in their work (Nielson, 2017). In each of these examples, the therapist is not constructed as administering the cure, instead the therapeutic encounter creates a set of conditions, which lead to an alternative state of consciousness and an opportunity for the patient's innate capacity for change to surface and unfold. It is the analyst or the mindfulness teacher's job to facilitate the optimum setting for the altered state to occur. However, since psychedelic therapy includes the presence of an actual psychoactive compound, training to provide it cannot be fully analogous to training to provide psychoanalysis or mindfulness-based interventions. At the same time the pronounced impact of extrapharmacological factors, such as set and setting, renders psychedelic therapy a poor parallel to pharmacological treatment with traditional psychotropic medications.

Training to provide both psychoanalysis and mindfulnessbased interventions includes direct experience of the method as a patient or yogi, respectively. The requirement of the personal psychoanalysis, or a training analysis, as a central part of psychoanalytic training has long been standard (Balint, 1954; Curtis \& Quaiser, 2005). Likewise, the researchers and practitioners who brought mindfulness from its Eastern origins into mainstream Western medicine carefully preserved the centrality of personal practice in the training process. In both instances, personal experience is an opportunity for the trainee to use the technique to study themselves as well as the method (Freud, 1970; Freudenberger \& Robbins, 1979; Kabat-Zinn, 2006). Mindfulness instructors teach out of their own "passion for the practice itself" (Kabat-Zinn, 2006, p. 150). Mindfulness instructors must develop an ongoing, carefully defined meditation practice before being accepted for formal instructor training programs and continue ongoing meditation practice to support their teaching and understanding of its healing potential (McCown et al., 2010), and the training analysis provides a platform for the analytic candidate to adopt and apply analytic perspectives and principals with both passion and discernment, as well as directly experience the patient role (Heimann, 1954). In contrast, no psychopharmacology researcher or psychiatric prescriber is either required or forbidden to have direct experience with any of the psychiatric medications they study or prescribe. Resolution of this conflict of paradigms is necessary for conceptualizing psychedelic research in a way that is not impoverished in the name of objectivity.

If the training analysis of psychoanalytic training is considered as a potential model, it is first necessary to clarify the nature of the training analysis. Historically, a training analysis was used to evaluate the analytic candidate: the analyst's approval was an integral part of the candidates' completion of the analytic training program (Curtis \& Quaiser, 2005). Contemporary analytic training institutions have shifted away from this model to analysis by a nonreporting analyst. As considerable period of analysis is still required, but complete confidentiality between candidate and analyst is maintained to save simple documentation of attendance (Curtis \& Quaiser, 2005). While the historical type of training analysis may more closely resemble shamanic training, we believe that the latter, more modern role of the non-reporting training analyst is a more appropriate potential model for psychedelic therapist training. Indeed, the MAPS MT-1 program is more closely related to the latter use of a training analysis as it is designed for educational purposes rather than evaluative.

Writing on the relevance of personal therapy for therapists in training has a long and interesting past. In continuing the discussion of its relevance to psychedelic therapist training, it is necessary to briefly explore the research on personal experience in preparing psychotherapists for their work. Using psychoanalytic training as a precedent, the idea that the personal experience is, can, or should be a part of preparation to become a psychotherapist is met with general acceptance in the field of psychotherapy training. However, quantitative empirical research has failed to consistently show a direct relationship between such experience and improved patient outcomes, perhaps because numerous confounds make studying the topic exceptionally difficult to quantify. On the other hand, qualitative studies consistently demonstrate a nuanced and dynamic relationship between the therapist's therapy and patient outcomes. For instance, MacDevitt (1987) studied 185 psychologists' responses to 25 hypothetical psychotherapy situations and found that the awareness of countertransference issues was significantly high and positively related to the amount of personal therapy a therapist had received. Gold and Hilsenroth (2009) conducted a prospective study comparing how two groups of therapists (half with prior experience in therapy and half without it) performed on outpatient assessments in a naturalistic setting. Significant differences were observed, with therapists having personal therapy experience trending toward having their patients remain in treatment longer and rating their alliance with their patients as stronger. However, the patients did not rate alliance with either therapist groups differently. Wheeler (1991) found that, among therapists who all had personal therapy, the more therapy a therapist had, the lower the rating of the quality of the alliance with their own patients. She speculates that this may be due to greater training leading to greater awareness of the complex, layered nature of therapeutic attachment.

Norcross, Strasusser-Kirtland, and Missar (1988) succinctly summarized six contributions of personal therapy to clinical practice: 
- Improvement of the emotional and mental functioning of the psychotherapist;

- More complete understanding of human relations and relative freedom from internal conflicts that impair treatment;

- Relief and care in response to the intense emotional demands of being a therapist;

- Aid in socialization into the healer role and direct experience of therapy's benefits;

- An improved, direct understanding of client's struggles and needs;

- Observing, first-hand, clinical methods and approaches, identification with their therapist's skills.

Taking these points back to our topic of psychedelic therapists, each seems relevant and reasonably likely to be helpful for psychedelic therapists in training, lending favor to the idea of including personal experience with the method even in the absence of a direct, quantifiable relationship to improved patient outcomes.

\section{HOW CURRENT PSYCHEDELIC RESEARCH ENGAGES IN PSYCHEDELIC THERAPIST TRAINING}

At present, training programs for therapists working in research studies of psilocybin in the USA exclude discussion of previous experience with psychedelics. Both the psilocybin-assisted treatment of alcohol-use disorder and MDMA-assisted treatment of PTSD protocols require therapists to have a background of clinical training and an experience in psychotherapy, as well as experience treating the specific diagnosis or problem addressed in each study. Therapists are provided with a detailed therapy manual and receive training in specific skills they are asked to employ (i.e., motivational interviewing), followed by recorded and fidelity monitored pilot-participant therapy sessions. Instruction is given on how to respond when participants experience difficult emotional states, how to demonstrate erratic behavior, or how to present with otherwise challenging reactions. For instance, in both cancer anxiety and alcohol study training, therapists are advised as to when and how to offer verbal or physical reassurance while simultaneously ensuring that the patient remains physically safe.

For the NYU Phase 2 Cancer Anxiety study (Ross et al., 2016), the therapy manual offered guidance to therapists on how to respond to questions from participants regarding their own psychedelic use. The guidance given was that such information should not be disclosed, but the manual suggests that therapists may indirectly allude to personal experience, or make disclosures limited to the use of psychedelics in legal settings (i.e., as part of a religious ceremony in South America). However, these disclosures are problematic as they may significantly affect the transference patterns that emerge. This may occur because patients, consciously or unconsciously, subscribe to the belief, outlined above, that a therapists' personal experience of psychedelic compounds is relevant to their skill and integrity in providing psychedelic therapy to others. (JG, who worked as a therapist on the cancer anxiety trial, reports that participants in that study frequently held this position.) This is a departure from the belief typically held by patients in traditional psychiatric settings that their prescribers' direct experience with psychiatric drugs, for instance SSRIs, is not relevant to their performing prescribing duties in an ethical, skillful manner.

An alternative approach is to acknowledge the importance of the question but not to answer it, instead exploring the fantasies, hopes, and fears that lie beneath the participant's question. This is the approach generally used in psychoanalytic therapy when a patient asks the therapist a question about the analysts' experience in their own treatments. However, present protocols for psychedelic therapy are short term and patients are unlikely to establish the level of trust with their therapists that would be required for meaningful exploration of their motivations for asking and fantasies about various possible answers.

The impact of sharing versus withholding such information on psychedelic therapy outcomes is a related question that has also not been systematically explored. It may seem intuitive that a participant would feel greater trust in a therapist who is experienced and comfortable with the terrain of psychedelic experiences. However, there are some potentially deeper complexities. For instance, a patient who imagines that his or her therapist has not had direct psychedelic experiences may question the therapists' motives and compassion, and this may lead to a failure of trust. Alternatively, a patient who imagines that his therapist has had her own psychedelic experiences may, in the absence of a firm understanding of the role of this experience in the therapists' training, imagine her to be irresponsible, untrustworthy, and engaged in dangerous and/or illicit activity. Such fantasies are commonly known as transference. The current moratorium on discussion of direct experiences with psychedelics prohibits the discussion and exploration of these issues with patients, the impact of which also deserves empirical exploration.

As noted above, an analytic candidate's personal experience of psychoanalysis is a key component of training. Although analysts usually speak very little of the content of their own personal analysis, it is axiomatic that the analyst draws on that direct experience of the method in developing his or her identity and in providing treatment to others. Similarly, those who teach meditation in therapeutic settings are expected to have and draw on personal experience with meditation as they offer themselves as teachers and guides of meditation as a therapy. It is from extensive personal experience that inspiration for meditation instruction comes, and it is from personal experience, as well as meditation teachings, that student's questions are answered. If the therapeutic action of psychedelic therapy is more akin to these examples than to a biologically conceptualized psychopharmacological treatment of psychiatric disorders, it is then particularly problematic that open discussion cannot occur as a part of the therapist training process.

\section{What makes therapists' personal experience with psychedelics difficult to discuss}

As we have described, the use of psychedelic medicines by those providing them to others for healing and personal 
growth in religious contexts, and therapists' personal experience with other therapies that employ alternative states of consciousness, are standard practice in each of those fields. Even so, it would not be defensible to simply presume that psychedelic clinical research must follow suit; there is theoretical and empirical work that need to be done on this question. There are two further key issues that must also be considered.

First, the result of the legal status of the psychedelic compounds currently being researched is that most therapists - including those who practice in federally sanctioned clinical research studies - now do not have legal access to these compounds for personal therapy or training purposes. Seeking and using any use of these compounds outside of the few sanctioned exceptions mentioned above would identify the researcher to colleagues, regulators, and funders as one who is engaged in illicit activity, to be of suspicious character, and stigmatized integrity, putting their ability to act responsibly when charged with clinical duties in question.

Second, the stigmata of hedonism and dangerousness remain attached to psychedelic use. Currently, the American mainstream associates psychedelics with 1960s counterculture use outside of medical settings that contributed to the passage of the Controlled Substances act of 1970 (Lander, 2014; Lattin, 2010). Although current research on psychedelics in academic settings follows rigorous regulatory guidelines, psychedelic therapy is often associated with the questionable behavior of some 1960s researchers regarding the widespread use outside of therapeutic and research settings, and unsubstantiated claims as to their safety and effects (Lattin, 2010). For this reason, today's clinical researchers, especially those who are working with psilocybin, create distance from many of our predecessors and cultivate an image of cautious, respectable, conscientious, and sober scientists (Langlitz, 2013). This distancing is understandable, significant problems did arise. However, we suggest that a thoughtful inquiry into the best training practices for psychedelic therapist must reopen the question of the role of personal experience, with all the concern for intellectual rigor and safety that are central to the current research.

A further challenge in discussing the potential value of personal experience with psychedelics may be located in a Western worldview that privileges ordinary waking consciousness above all others. This worldview often marginalizes and devalues healing methods that involve ecstatic or highly aroused emotional/cognitive states (Walsh \& Grob, 2006). Psychedelic therapy questions the position that meaningful, profound change can occur only in sober consciousness, and that knowledge and growth found in an alternative state of consciousness - particularly one occasioned by a psychedelic compound - are suspect. When induced by a psychedelic, such states of consciousness may be disregarded as pathological (evoking a pathological regression or psychotic states), trivial (pleasurable but meaningless), or dangerous (signs of addiction or uncontrollable destructive behavior). From this follows doubt and skepticism that a therapist's knowledge and understanding derived in such states could be reliable, valuable, and even essential for performing clinical work with these compounds in a responsible and skillful manner.

\section{What, when, and why? Issues for future research}

Personal use of psychedelics by researchers and clinicians played a central role in the rise and fall of the first wave of psychedelic research. It was one of the initial indications for administering LSD and later, a point used to discredit psychedelic researchers and their findings. The role of personal experience in the second wave of psychedelic research is largely undocumented and unknown, but it is likely to have some influence at minimum, personal use may impact research design, therapy outcomes, and objectivity. In light of recent promising clinical findings and strict adherence to rigorous scientific research methods among today' researchers, we propose that scientific inquiry into direct experience is now feasible, timely, and necessary.

Any discussion of the role of personal experience with psychedelics in psychedelic therapist training and development must be undertaken with caution. The vibrant progress of sanctioned research on psilocybin and MDMA in the past 25 years has made rescheduling in the near future a possibility. The field does well to stay focused on the projects and outcomes that will eventually help realize this goal. Any research on the experiences of psychedelic therapists themselves needs to maintain a balanced approach in understanding the role of such experience, without reliance on conjecture, deeply held opinion, or what has traditionally been done in religious or underground contexts.

To this end, we propose the following research initiatives to investigate the role of psychedelic therapists' personal use of psychedelics in training and professional development.

First, a thorough analysis of the data collected on the Spring Grove research group's provision of LSD to researchers and therapists between 1969 and 1974 is in order, as this may offer insight into the role of these experiences for therapist and researcher development at this point in history. EMN is currently undertaking this project with expected completion in 2018.

Second, analysis of the experiences of the MAPS PTSD therapists who have received MDMA through the MT-1, or future, similar programs, should be conducted to explore the impact of this experience on therapist development and patient outcomes.

Third, we suggest a study designed to answer research questions on the relationship of direct experience to identity formation, therapeutic alliance, and clinical outcomes by giving a course of psilocybin-assisted psychotherapy to therapists who work with psilocybin as a part of their training and measuring these constructs empirically. Although this research would require a substantial allocation of resources, this initiative could explore, in a rigorous way, how study therapists apply their experience in their subsequent work, allowing for evidence-based improvement in training methods and a clearer understanding of the role of personal experience with psilocybin in therapeutic setting on therapist development. Additional/alternative research projects could focus on the role of personal experience with psilocybin and other psychedelics in non-clinical settings and in alternative states of consciousness achieved by other, non-pharmacological means.

We close by restating our empirical question, one that is potentially critical to the continued advancement of 
psychedelic therapy research: Does psychedelic therapists' personal experience with psychedelics affect the outcomes of the patients they treat and of so, how? Research to answer this question should be conducted in accordance with the highest standards of research design, to ensure that it is not biased by preexisting beliefs and suppositions. Findings should be used to guide the development of training programs and decisions regarding the inclusion of psychedelic experiences for therapists in training. Given the persistence of this question, attempts to answer it with first-wave psychedelic research, comparison with psychedelic use in other contexts, or drawing on other forms of psychotherapy as models are no longer sufficient.

Acknowledgements: EMN's work on this manuscript was supported by the National Institute on Drug Abuse Grant T32DA007233-33S1 while the author was a postdoctoral fellow at NYU Rory Meyers College of Nursing. Opinions are those of the authors and do not represent the official views of National Institutes of Health, NIDA, or NYU. The authors would like to thank Ingmar Gorman, Kelley O’Donnell, Stephen Ross, Jeremy Safran, Adrienne Harris, and Tehseen Noorani for their valuable feedback on early versions of this manuscript.

Conflict of interest: The authors declare no conflict of interest.

\section{REFERENCES}

Abramson, H. (1976). The use of LSD in psycotherapy and alcoholism. New York, NY: Bobbs-Merrill Company.

Ayahuasca Foundation. (2017). The Ayahuasca Foundation. Retrieved July 2, 2017, from https://www.ayahuascafoundation. org/ayahuasca-foundation-about-us/

Balint, M. (1954). Analytic training and training analysis. The International Journal of Psycho-Analysis, 35, 157.

Blewett, D. B., \& Chwelos, N. (1959). Handbook for the therapeutic use of lysergic acid diethylamide-25: Individual and group procedures. Santa Cruz, CA: MAPS.

Bogenschutz, M. P., \& Forcehimes, A. A. (2016). Development of a psychotherapeutic model for psilocybin-assisted treatment of alcoholism. Journal of Humanistic Psychology, 57(4), 389-414. doi:10.1177/0022167816673493

Bogenschutz, M. P., Forcehimes, A. A., Pommy, J. A., Wilcox, C. E., Barbosa, P. C. R., \& Strassman, R. J. (2015). Psilocybinassisted treatment for alcohol dependence: A proof-of-concept study. Journal of Psychopharmacology, 29(3), 289-299. doi:10.1177/0269881114565144

Curtis, R. C., \& Quaiser, M. (2005). Training analyses: Historical considerations and empirical research. In J. D. Geller, J. C. Norcross, \& D. E. Orlinsky (Eds.), Psychotherapist's own psychotherapy: Patient and clinician perspectives. New York, NY: Oxford University Press.

Fernandez, J. W., \& Fernandez, R. L. (2001). The use of iboga [ine] in an equatorial African ritual context and the binding of time, space, and social relationships (Vol. 56, pp. 235-249). New York, NY: Academic Press.

Frederking, W. (1955). Intoxicant drugs (mescaline and lysergic acid diethylamide) in psychotherapy. Journal of Nervous \&
Mental Disease, 121(3), 262-266. doi:10.1097/00005053195503000-00010

Freud, A. (1970). Problems of training analysis [Abstract]. Psyche: Zeitschrift für Psychoanalyse und ihre Anwendungen, 24(8), 565-576.

Freud, S. (1912). Recommendations to physicians practicing psycho-analysis. In J. Strachey (Ed.), The case of Schreber, papers on technique and other works (Vol. XII (1911-1913), pp. 109-120). London: The Hogarth Press and the Institute of Psycho-analysis.

Freudenberger, H. J., \& Robbins, A. (1979). The hazards of being a psychoanalyst. Psychoanalytic Review, 66(2), 275.

Garcia-Romeu, A., Kersgaard, B., \& Addy, P. H. (2016). Clinical applications of hallucinogens: A review. Experimental and Clinical Psychopharmacology, 24(4), 229-268. doi:10.1037/ pha0000084

Gold, S. H., \& Hilsenroth, M. J. (2009). Effects of graduate clinicians' personal therapy on therapeutic alliance. Clinical Psychology \& Psychotherapy, 16(3), 159-171. doi:10.1002/ cpp.612

Green, H., Barkham, M., Kellett, S., \& Saxon, D. (2014). Therapist effects and IAPT Psychological Wellbeing Practitioners (PWPs): A multilevel modelling and mixed methods analysis. Behaviour Research and Therapy, 63, 43-54. doi:10.1016/j. brat.2014.08.009

Griffiths, R. R., Johnson, M. W., Carducci, M. A., Umbricht, A., Richards, W. A., Richards, B. D., Cosimano, M. P., \& Klinedinst, M. A. (2016). Psilocybin produces substantial and sustained decreases in depression and anxiety in patients with life-threatening cancer: A randomized double-blind trial. Journal of Psychopharmacology, 30(12), 1181-1197. doi:10.1177/0269881116675513

Grob, C. S., Danforth, A. L., Chopra, G. S., Hagerty, M., McKay, C. R., Halberstadt, A. L., \& Greer, G. R. (2011). Pilot study of psilocybin treatment for anxiety in patients with advancedstage cancer. Archives of General Psychiatry, 68(1), 71-78. doi:10.1001/archgenpsychiatry.2010.116

Grof, S. (2008). LSD psychotherapy: The healing potential of psychedelic medicine. Ben Lomond, CA: MAPS.

Halberstadt, N. (2014). MDMA-assisted psychotherapy: A view from both sides of the couch. Maps Bulletin (Special Edition), 24(1), 4-6.

Hartogsohn, I. (2016). Set and setting, psychedelics and the placebo response: An extra-pharmacological perspective on psychopharmacology. Journal of Psychopharmacology, 30(12), 1259-1267. doi:10.1177/0269881116677852

Heimann, P. (1954). Problems of the training analysis. The International Journal of Psycho-Analysis, 35, 163.

Hofmann, A. (2005). LSD, my problem child: Reflections on sacred drugs, mysticism, and science. Santa Cruz, CA: Multidisciplinary Association for Psychedelic Studies.

Hyde, R. W. (1960). Psychological and social determinants of drug action. In G. J. Sarwer-Foner (Ed.), The dynamics of psychiatric drug therapy (pp. 297-315). Springfield, IL: Thomas.

Johnson, M. W., Garcia-Romeu, A., Cosimano, M. P., \& Griffiths, R. R. (2014). Pilot study of the 5-HT2AR agonist psilocybin in the treatment of tobacco addiction. Journal of Psychopharmacology, 28(11), 983-992. doi:10.1177/0269881114548296

Kabat-Zinn, J. (2006). Mindfulness-based interventions in context: Past, present, and future. Clinical Psychology: Science and Practice, 10(2), 144-156. doi:10.1093/clipsy.bpg016 
Kurland, A. A., Savage, C., Pahnke, W., Unger, S., \& Grof, S. (1969). Application to amend IND-3250 for the administration of LSD for training purposes. Research Proposal.

Lander, D. R. (2014). "Legalize Spiritual Discovery": The trials of Dr. Timothy Leary. In B. C. Labate \& C. Cavnar (Eds.), Prohibition, religious freedom, and human rights: Regulating traditional drug use (pp. 165-187). Berlin, Heidelberg: Springer.

Langlitz, N. (2013). Neuropsychedelia: The revival of hallucinogen research since the decade of the brain. Berkeley, CA: University of California Press.

Larrison, C. R., Schoppelrey, S. L., Hack-Ritzo, S., \& Korr, W. S. (2011). Clinician factors related to outcome differences between black and white patients at CMHCs. Psychiatric Services, 62(5), 525-531. doi:10.1176/ps.62.5.pss6205_0525

Lattin, D. (2010). The Harvard psychedelic club: How Timothy Leary, Ram Dass, Huston Smith, and Andrew Weil killed the fifties and ushered in a new age for America. New York, NY: HarperOne.

Leary, T., Metzner, R., \& Alpert, R. (2000). The psychedelic experience: A manual based on the Tibetan book of the dead. New York, NY: Citadel Press, Kensington Publishing Corp.

MacDevitt, J. W. (1987). Therapists' personal therapy and professional self-awareness. Psychotherapy: Theory, Research, Practice, Training, 24(4), 693-703. doi:10.1037/h0085769

Mangini, M. (1998). Treatment of alcoholism using psychedelic drugs: A review of the program of research. Journal of Psychoactive Drugs, 30(4), 381-418. doi:10.1080/02791072. 1998.10399714

MAPS. (2010). Training protocol for MDMA-assisted psychotherapy researchers (Open Label Phase 1 Study). Retrieved from http://www.maps.org/research/mdma/mdma-research-timeline/ 355-training-protocol-for-mdma-ptsd-researchers-phase-1

McCown, D., Reibel, D., \& Micozzi, M. S. (2010). Teaching mindfulness: A practical guide for clinicians and educators. New York, NY: Springer.

Metzner, R. (1998). Hallucinogenic drugs and plants in psychotherapy and shamanism. Journal of Psychoactive Drugs, 30(4), 333-341. doi:10.1080/02791072.1998.10399709

Mithoefer, M. C., Wagner, M. T., Mithoefer, A. T., Jerome, L., \& Doblin, R. (2011). The safety and efficacy of $\pm 3,4$ methylenedioxymethamphetamine-assisted psychotherapy in subjects with chronic, treatment-resistant posttraumatic stress disorder: The first randomized controlled pilot study. Journal of Psychopharmacology, 25(4), 439-452. doi:10.1177/ 0269881110378371

Nickles, D. (2015). Criminals and researchers: Perspectives on the necessity of underground research. Paper presented at the Breaking Convention: 3rd International Conference on Psychedelic Consciousness, University of Greenwich, London.

Nielson, E. (2017). Unpublished Interview.

Norcross, J., Strasusser-Kirtland, D., \& Missar, D. (1988). The processes and outcomes of psychotherapists' personal treatment experiences. Psychotherapy, 25(1), 36-43. doi:10.1037/ h0085321

Oram, M. (2012). Efficacy and enlightenment: LSD psychotherapy and the drug amendments of 1962. Journal of the History of Medicine and Allied Sciences, 69(2), 221-250. doi:10.1093/ jhmas/jrs050
Osório, F. D. L., Sanches, R. F., Macedo, L. R., dos Santos, R. G., Maia-de-Oliveira, J. P., Wichert-Ana, L., Araujo, D. B., Riba, J., Crippa, J. A., \& Hallak, J. E. (2015). Antidepressant effects of a single dose of ayahuasca in patients with recurrent depression: A preliminary report. Revista Brasileira de Psiquiatria, 37(1), 13-20. doi:10.1590/1516-4446-2014-1496

Ross, S., Bossis, A., Guss, J., Agin-Liebes, G., Malone, T., Cohen, B., Mennenga, S. E., Belser, A., Kalliontzi, K., Babb, J., Su, Z., Corby, P., \& Schmidt, B. L. (2016). Rapid and sustained symptom reduction following psilocybin treatment for anxiety and depression in patients with life-threatening cancer: A randomized controlled trial. Journal of Psychopharmacology, 30(12), 1165-1180. doi:10.1177/0269881116675512

Rubin, J. B. (1985). Meditation and psychoanalytic listening. Psychoanalytic Review, 72(4), 599.

Sessa, B., \& Fischer, F. M. (2016). Underground MDMA-, LSD- and 2-CB-assisted individual and group psychotherapy in Zurich: Outcomes, implications and commentary. Drug Science, Policy and Law, 2, 1-8. doi:10.1177/2050324 515578080

Smart, R. G., Storm, T., Baker, E. F., \& Solursh, L. (1966). A controlled study of lysergide in the treatment of alcoholism. 1. The effects on drinking behavior. Quarterly Journal of Studies on Alcohol, 27(3), 469-482.

Strassman, R. (2001). DMT: The spirit molecule. Rochester, VT: Park Street Press.

Thomas, N., \& Humphrey, C. (1996). Shamanism, history, and the state. Ann Arbor, MI: University of Michigan Press.

Walsh, R., \& Grob, C. S. (2006). Early psychedelic investigators reflect on the psychological and social implications of their research. Journal of Humanistic Psychology, 46(4), 432-448. doi:10.1177/0022167806286745

Wheeler, S. (1991). Personal therapy: An essential aspect of counsellor training, or a distraction from focusing on the client? International Journal for the Advancement of Counselling, 14(3), 193-202. doi:10.1007/BF00119182

White, W. L. (2013). A life in addiction psychiatry: An interview with Dr. Herb Kleber. Retrieved from http:// www.williamwhitepapers.com/pr/2013\%20Dr.\%20\%20Herb $\% 20$ Kleber.pdf

Winkelman, M. J. (2007). Shamanic guidelines for psychedelic medicine. In M. J. Winkelman \& T. B. Roberts (Eds.), Psychedelic medicine: New evidence for hallucinogenic substances as treatments (Vol. 2, pp. 143-168). Westport, CT: Praeger Publishers.

Winkler, P., \& Csémy, L. (2014). Self-experimentations with psychedelics among mental health professionals: LSD in the former Czechoslovakia. Journal of Psychoactive Drugs, 46(1), 11-19. doi:10.1080/02791072.2013.873158

Winkler, P., Gorman, I., \& Kočárová, R. (2016). Chapter 72 - Use of LSD by mental health professionals A2. In V. R. Preedy (Ed.), Neuropathology of drug addictions and substance misuse (pp. 773-781). San Diego, CA: Academic Press.

Yensen, R., \& Dryer, D. (1992). Thirty years of psychedelic research: The Spring Grove experiment and its sequels. Paper presented at the European College of Consciousness (ECBS) International Congress, Goettingen, Germany. 